\title{
Adipose Extracellular Vesicles in Intercellular and Inter-Organ Crosstalk in Metabolic Health and Diseases
}

\author{
Zhe Huang ${ }^{1,2}$ and Aimin $\mathrm{Xu}^{1,2,3 *}$ \\ ${ }^{1}$ The State Key Laboratory of Pharmaceutical Biotechnology, The University of Hong Kong, Hong Kong, China, ${ }^{2}$ Department \\ of Medicine, The University of Hong Kong, Hong Kong, China, ${ }^{3}$ Department of Pharmacology and Pharmacy, The University \\ of Hong Kong, Hong Kong, China
}

\section{OPEN ACCESS}

Edited by:

Amaia Rodríguez,

University of Navarra, Spain

Reviewed by:

Sara Becerril,

University of Navarra, Spain

Tania Romacho,

German Diabetes Center

(DDZ), Germany

${ }^{*}$ Correspondence:

Aimin Xu

amxu@hku.hk

Specialty section:

This article was submitted to

Inflammation,

a section of the journal

Frontiers in Immunology

Received: 21 September 2020

Accepted: 05 February 2021

Published: 25 February 2021

Citation:

Huang Z and Xu A (2021) Adipose

Extracellular Vesicles in Intercellular and Inter-Organ Crosstalk in Metabolic

Health and Diseases.

Front. Immunol. 12:608680.

doi: 10.3389/fimmu.2021.608680
Adipose tissue (AT) is a highly heterogeneous and dynamic organ that plays important roles in regulating energy metabolism and insulin sensitivity. In addition to its classical roles in nutrient sensing and energy storage/dissipation, AT secretes a large number of bioactive molecules (termed adipokines) participating in immune responses and metabolic regulation through their paracrine and/or endocrine actions. Adipose-derived extracellular vesicles (ADEVs), including exosomes, microvesicles (MVs), and apoptotic bodies, have recently emerged as a novel class of signal messengers, mediating intercellular communications and inter-organ crosstalk. In AT, ADEVs derived from adipocytes, immune cells, mesenchymal stem cells, endothelial cells are actively involved in modulation of immune microenvironment, adipogenesis, browing of white adipose tissue, adipokine release and tissue remodeling. Furthermore, ADEVs exert their metabolic actions in distal organs (such as liver, skeletal muscle, pancreas and brain) by sending genetic information (mainly in the form of microRNAs) to their target cells for regulation of gene expression. Here, we provide an updated summary on the nature and composition of ADEVs, and their pathophysiological functions in regulating immune responses, whole-body insulin sensitivity and metabolism. Furthermore, we highlight the latest clinical evidence supporting aberrant production and/or function of ADEVs as a contributor to obesity-related chronic inflammation and metabolic complications and discuss the opportunities and challenges in developing novel therapies by targeting ADEVs.

Keywords: exosome, microRNA, adipose tissue macrophage, metabolic homeostasis, inflammation, cell-cell communication

\section{INTRODUCTION}

Obesity, characterized by excessive accumulation of adipose tissue (fat), is a highly complex multifaceted chronic disease and one of the major risk factors for a cluster of cardio-metabolic diseases, including type 2 diabetes (T2D), dyslipidemia, non-alcoholic fatty liver disease (NAFLD), hypertension, coronary heart disease and stroke (1). Furthermore, obesity-related complications are the most commonly reported underlying conditions that predispose individuals with viral infections, including the current coronavirus disease (COVID-19) to severe outcomes (2). 
Adipose tissue is a complex metabolic organ with profound effects on the regulation of systemic metabolism and maintenance of energy homeostasis. In addition to its classical role in nutrient handling, including energy storage in the form of triglycerides during feeding and releasing of free fatty acids (FFAs) during fasting, adipose tissue also serves as an active endocrine organ secreting a variety of adipokines, which are bioactive peptides including cytokines, peptide hormones and enzymes acting in an autocrine, paracrine or endocrine manner to regulate energy metabolism, immune responses and cardiovascular homeostasis. Adipokines have been shown to modulate adipogenesis, adipocyte metabolism, and immune cell infiltration locally within adipose tissue. Additionally, they can exert their biological effects in distal organs to maintain systemic energy homeostasis and insulin sensitivity $(3,4)$. However, during obesity, adipose tissue undergoes unhealthy expansion leading to numerous detrimental consequences, including dysregulated secretion of adipokines, hypoxia, cell death and altered immune microenvironment which give rise to adipose inflammation (5). Unresolved chronic inflammation in adipose tissue is a major contributor to systemic lowgrade inflammation and has been reported as a culprit in obesity-related comorbidities. The first evidence for adipose inflammation at the interface between obesity and metabolic dysregulation was provided by studies demonstrating increased production and secretion of the inflammatory cytokine tumor necrosis factor alpha $(\mathrm{TNF} \alpha)$ from adipose tissues in obese rodents and human subjects $(6,7)$. Neutralization of TNF $\alpha$ in obese rats counteracted diet-induced insulin resistance and glucose intolerance (7). Subsequent studies reported that selective inactivation of pro-inflammatory signaling pathways in adipose tissue by inhibition of key signaling molecules, including c-Jun N-terminal kinase (JNK) and nuclear factor- $\kappa \mathrm{B}$ $(\mathrm{NF}-\kappa \mathrm{B})$ disrupted the link between obesity and metabolic dysregulation (8-10).

In addition to the classical polypeptide adipokines and cytokines, various types of cells in adipose tissue also produce and release extracellular vesicles (EVs) including exosomes with a diameter of $30-100 \mathrm{~nm}$ originated from cytoplasmic multivesicular bodies that fuse with the plasma membrane and microvesicles (MVs) that are $100-1000 \mathrm{~nm}$ in diameter and released directly from the plasma membrane into the extracellular space. Both types of such adipose-derived extracellular vesicles (ADEVs) are similar to the original cells in composition, transporting bioactive molecules, including proteins, lipids, and nucleic acids to their target cells within adipose tissue or in distant organs, therefore mediating intercellular and interorgan crosstalk. A growing body of evidence suggest that ADEVs play important roles in the regulation of metabolic inflammation, energy metabolism and insulin sensitivity (11-15). Altered abundance or content of ADEVs may be causally linked to obesity-related metabolic complications.

In this review, we summarize the nature and compositions of ADEVs derived from different cellular origins in adipose tissue and their roles as local and/or distal signaling mediators in regulating metabolic homeostasis. Furthermore, we highlight the latest evidence for the clinical association of aberrant production and/or functions of ADEVs with various obesity-related metabolic disorders, and discuss the therapeutic potentials of targeting ADEVs for the treatment of obesity-related metabolic complications and challenges in this field.

\section{ADIPOSE TISSUE IN HEALTH AND METABOLIC DISEASES}

\section{Heterogeneity of Adipose Tissue}

Adipose tissue in mammals is categorized into two main types, white adipose tissue (WAT) and brown adipose tissue (BAT). WAT mainly consists of white adipocytes, which contain a single large lipid droplet (referred to as unilocular lipid structure) and few mitochondria, thus is a primary site for energy storage. White adipocytes are highly responsive to hormones such as insulin to take up and store nutrients in the form of triglycerides after food ingestion. They also respond to biogenic amines such as catecholamines to supply energy in the forms of FFAs and glycerol during nutrient deprivation (5). WAT is distributed throughout the body. Main depots include subcutaneous adipose tissue (SAT), which is beneath the skin storing more than $80 \%$ of total fat in the body and is mainly located in the abdominal and gluteofemoral regions in humans or between the scapulae and in the inguinal region spreading from the dorsolumbar to the gluteal region in rodents, and visceral adipose tissue (VAT), which stores $5-20 \%$ of total body fat and is associated with internal organs mainly in perigonadal, mesenteric, retroperitoneal, epicardial and periadventitial regions in rodents and humans $(16,17)$. In addition, there are also small adipose depots including epicardial and intermuscular adipose tissue with specialized functions related to cardiovascular system or skeletal muscle (17). While the major function of SAT is to store excess energy in response to energy surplus and is therefore considered as beneficial, VAT is more closely linked to adverse metabolic profile and inflammation in obese subjects $(18,19)$.

BAT mainly consists of brown adipocytes with multilocular lipid droplets and a large number of highly oxidative, naturally uncoupled mitochondria, and is important for the regulation of body temperature through non-shivering thermogenesis. The thermogenic capacity of brown adipocytes is primarily attributed to the mitochondrial inner membrane protein, uncoupling protein-1 (UCP1), which catalyzes a proton leak across the inner mitochondrial membrane, thus uncouples oxidative phosphorylation from ATP synthesis, and converts chemical energy to heat $(20,21)$. Brown adipocytes are located in the well-defined anatomical BAT depots such as interscapular, peri-aortic, intercostal and mediastinal regions of rodents. In addition to the classical brown adipocytes, beige adipocytes also contribute to thermogenesis. Although beige adipocytes share similar morphological characteristics and thermogenic capacity with classical brown adipocytes, they arise from different precursor cells $(22,23)$. In humans, although early studies suggested that BAT is present only in neonates to prevent from hypothermia resulted from high body surface area-to-mass ratio, recent positron emission tomography coupled with computer 
tomography (PET/CT)-based approaches have identified the existence of metabolically-active BAT in the supraclavicular, ventral cervical and thoracic regions of adults (24-26). While the predominant form of the interscapular BAT in human neonates is classical brown adipocytes, BAT in human adults share more molecular features with beige adipocytes (23). Furthermore, both amount and activity of BAT in adults is negatively correlated with body weight, T2D and cardiovascular events, but positively correlated with energy expenditure $(24,25,27)$.

\section{Cellular Composition of Adipose Tissue}

Although adipocytes are the dominant cell type in adipose tissue, there are also non-adipocyte compartment named as stromal vascular fraction (SVF), which include preadipocytes, adipose tissue-derived stem cells (ADSCs), endothelial cells, pericytes, and various immune cells. Preadipocytes can be differentiated into mature adipocytes to regulate adipogenesis and WAT expansion (28). ADSCs undergo self-renewal and are multipotent, with the potential to differentiate into numerous cell types, including adipogenic lineages, endothelial cells, osteoblasts, chondrocytes and myocytes (29). Endothelial cells and pericytes provide vasculature to adipose tissue by forming capillaries (22, 30-33). Presence of immune cells was not realized till discovery of adipose-resident macrophages responsible for producing pro-inflammatory cytokines in obese mice and humans in the early 2000s $(34,35)$. It is now known that adipose tissue is home to both innate immune cells such as macrophages, neutrophils, eosinophils and dendritic cells and adaptive immune cells, including $\mathrm{T}$ cells and $\mathrm{B}$ cells, which collaboratively play important roles in clearance of apoptotic cells, maintenance of adipose tissue function and homeostasis (36).

\section{Adipose Tissue Inflammation as a Culprit in Obesity-Related Disorders}

A growing body of evidence suggests that chronic inflammation in adipose tissue, characterized by infiltration of proinflammatory immune cells and aberrant production of adipokines, is a major contributor to obesity-induced systemic inflammation, insulin resistance and metabolic dysregulation $(37,38)$. Obesity leads to an expansion of adipose tissue driven by adipocyte hyperplasia and hypertrophy. The lipid-laden adipocytes in obesity undergo necrosis and/or apoptosis, leading to aberrant production of adipokines and altered cellular composition in adipose tissue (39). In obesity, the hypertrophic adipocytes exhibit impaired secretion of anti-inflammatory adipokines such as adiponectin, but augmented secretion of a large number of pro-inflammatory mediators, such as IL-6, C-C motif chemokine ligand 2 (CCL2), IL-1 $\beta$ and resistin that lead to a chronic inflammatory state linking obesity to its cardiometabolic comorbidities including insulin resistance, T2D and cardiovascular events (40).

During the progression of obesity, expansion of adipose tissue also causes infiltration and activation of immune cells involved in both innate and adaptive immunity, which in turn trigger a series of inflammatory responses within the tissue. Among adipose-resident immune cells, macrophages are the most abundant cell type, accounting for $40-50 \%$ of total cells of adipose tissue in obese humans (41). In obese adipose tissues, macrophages form crown-like structures (CLSs) surrounding dying or dead adipocytes. The number of adipose tissue-resident macrophages (ATMs) is closely associated with the magnitude of insulin resistance and metabolic perturbance, whereas selective depletion of ATMs by genetic or pharmacological approaches is sufficient to prevent obesity-related insulin resistance and metabolic complications in obese mice $(34,42)$. Macrophages are highly plastic in nature, exhibiting different phenotypes ranging from the classically activated, pro-inflammatory M1 to alternatively activated, anti-inflammatory M2 in response to changing environment (41). The lean adipose tissue is dominated by M2 macrophages which plays an important role in maintaining the tissue homeostasis through phagocytosis of dead adipocytes, secretion of anti-inflammatory cytokines and other regulatory factors for angiogenesis, adipogenesis, and regulation of adaptive thermogenesis (43). However, obesity causes a striking phenotypic change of ATMs from the antiinflammatory M2 toward the pro-inflammatory M1, the latter of which produce pro-inflammatory cytokines to exacerbate metabolic inflammation and insulin resistance $(41,43)$. However, the precise mechanisms whereby adipocyte and various immune cells crosstalk with each other to aggravate obesity-induced adipose inflammation and metabolic dysregulation remain poorly defined.

\section{CELLULAR ORIGIN OF ADEVs AND THEIR ROLES IN CELL-CELL COMMUNICATIONS}

EVs are enclosed by a lipid bilayer and classified into three main classes, including exosomes, MVs and apoptotic bodies (44). Exosomes are a homogenous population of EVs at $30-100 \mathrm{~nm}$ in diameter. Biogenesis of exosomes begins from endocytosismediated invagination of the plasma membrane, resulting in endocytotic vesicles, which are subsequently transported to the early endosomes. Membranes of the endosomes are budded into the lumen to form intraluminal vesicles (ILVs) or multivesicular bodies (MVBs). MVBs can fuse with lysosomes for degradation or with the plasma membrane to release the internal vesicles into extracellular space as exosomes (45). Exosomes show the same orientation with the plasma membrane composed of a lipid bilayer with extracellular domains of proteins exposed at the surface. The lipid bilayer of exosomes encloses a droplet of cytoplasm containing various types of molecules including nucleic acids, proteins and lipids (45). Cells can also produce MVs with heterogenous populations ranging from 100 to $1,000 \mathrm{~nm}$ in diameter. In contrast to exosomes derived from the endolysosomal pathway, MVs are formed by direct budding and shedding from the plasma membrane (44). EVs released from cells undergoing apoptosis are referred to as apoptotic bodies with a diameter of $1,000-5,000$ $\mathrm{nm}$ (44).

Multiple types of cells in adipose tissue, including adipocytes, macrophages, ADSCs and endothelial cells are known to secrete EVs, which in turn, act in a paracrine or endocrine manner to mediate intercellular and inter-organ crosstalk in modulation 


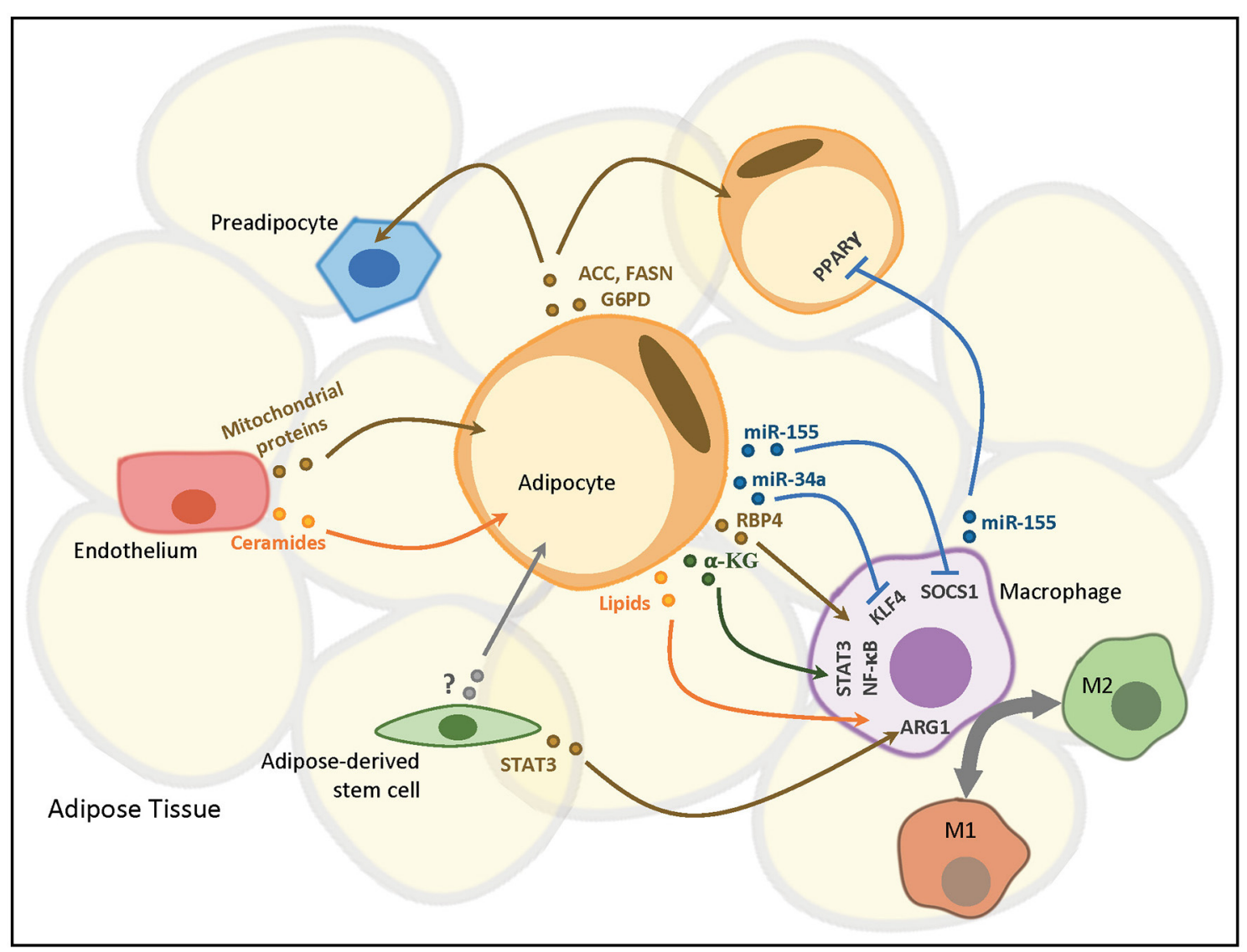

FIGURE 1 | ADEVs-mediated intercellular communication in adipose tissue. Adipocytes mediate the polarization and immunomodulatory responses of adipose-resident macrophages (ATMs) in a paracrine manner via various vesicular components. ATMs reciprocally regulate adipocyte insulin sensitivity by releasing miRNA-containing EVs to adipocytes. Adipocytes also delivery exosomal proteins to neighboring preadipocytes and adipocytes in both a paracrine and an autocrine manner, respectively, to modulate lipogenesis. Adipose-derived stem cells (ADSCs) confer EV-mediated paracrine effects on both adipocytes and ATMs to regulate adipocyte reprogramming and macrophage polarization, respectively. Endothelial cells in adipose tissue transfer EVs containing proteins and lipids capable of modulating cellular signaling pathways to adipocytes. ACC, acetyl-CoA carboxylase; FASN, fatty acid synthase; G6PD, glucose-6-phosphate dehydrogenase; PPAR $\gamma$, peroxisome proliferator activated receptor gamma; RBP4, retinol binding protein 4; $\alpha$-KG, $\alpha$-ketoglutarate; SOCS1, suppressor of cytokine signaling 1; KLF4, krüppel-like factor 4; STAT3, signal transducer and activator of transcription 3; NF-кB, nuclear factor kappa B; ARG1, arginase 1.

of adipose tissue and systemic homeostasis (Figure 1), as detailed below.

\section{Adipocytes}

The presence of EVs in the culture medium of adipose tissue explants has been demonstrated in both mouse and human studies $(46,47)$. Further analysis of EVs isolated from ex vivo human adipose tissue explant cultures has identified both adiponectin-positive and adiponectin-negative subsets by differential ultracentrifugation combined with immunoblotting analysis (47). Since adiponectin is expressed predominantly in adipocytes, the adiponectin-positive EVs were suggested to be derived from adipocytes. In agreement with this notion, certain portion of exosomes isolated from mouse serum has also been demonstrated to contain adiponectin and low level of resistin (48). However, as detection of adiponectin in EVs was achieved by immunoblotting, whether adiponectin is located on the surface or inside of adipocyte-derived EVs remains unknown.
The secretion of EVs from adipocytes has been further confirmed by in-vitro cultures of rat primary adipocytes or adipocytes differentiated from mouse 3T3-L1 pre-adipocytes and human Simpson Golabi Behmel Syndrome (SGBS) pre-adipocytes (47, 49). In addition to adiponectin and resistin, there are several other adipocyte-specific proteins have been identified as the markers of adipocyte-derived EVs, including perilipin A and fatty acid binding protein 4 (FABP4) $(50,51)$.

Although adipocyte-derived exosomes account for a minority of circulating exosomes under normal condition (52), production of ADEVs can vary under different conditions. For example, the circulating level of lipid-filled vesicles derived from adipocytes was increased by approximately 2-folds in obese mice vs. lean animal (52). Similarly, the number of exosomes isolated from VAT was elevated in human patients with insulin resistance (47). Under chronic cold exposure, the number of exosomes released from explants isolated from both interscapular BAT and inguinal WAT 
of mice was significantly induced. In-vitro studies showed that release of exosomes from beige and brown adipocytes was increased by treatment with cAMP, which is the second messenger induced by cold exposure or beta-adrenergic stimulation (53).

Adipocyte-derived EVs confer in part the paracrine interaction between adipocytes and macrophages. EVs released from human adipocyte culture were able to induce differentiation of monocytes into ATM-like macrophages in vitro. Adiponectin-positive EVs from human adipose tissue explants were more potent than the adiponectin-negative subset in promoting monocyte differentiation into ATMs as they induced the expression of mixed pro- and anti-inflammatory markers, which are characteristic of ATMs, in monocytes in vitro (47). Furthermore, adipocyte-derived EVs isolated from high-fat diet (HFD)-fed mice drove polarization of macrophages toward the pro-inflammatory M1 phenotypes in bone marrow-derived macrophages (BMDMs) in vitro by miR-155, which inhibited the expression of suppressor of cytokine signaling 1 (SOCS1), leading to suppression of signal transducer and activator of transcription 6 (STAT6) (54). In addition to adipose tissue, adipocytes also exist in tumor microenvironment, including melanoma. It is reported by recent studies that adipocytesecreted exosomes were taken up by tumor cells, resulting in increased melanoma migration and invasion through fatty acid oxidation. Such effects were amplified in obese animals $(55,56)$.

\section{Macrophages}

In addition to adipocytes, ATMs also produce EVs to modulate inflammatory responses and metabolic homeostasis. In vitro, secretion of EVs was detected in the culture medium of human THP-1-derived macrophages $(14,57)$. These EVs were identified as exosomes with a diameter of 30$100 \mathrm{~nm}$ by using transmission electron microscopy (14). The macrophage-derived exosomes can be effectively internalized into adipocytes. Exosomes from LPS-activated macrophages promote the expression of inflammation-related genes in adipocytes (57). Interestingly, when THP- 1 monocytes-derived macrophages are polarized to M1 or M2 phenotype by LPS plus IFN- $\gamma$ or IL-4, respectively, exosomes derived from M1 macrophages impair insulin signaling in human adipocytes, while the M2 macrophage-derived exosomes enhance insulin signaling and glucose uptake in adipocytes (14). Likewise, EVs harvested from ATMs isolated from VAT of mice were also found to be exosomes as they are $30-100 \mathrm{~nm}$ in size (13). It is further evidenced by detection of exosomal membrane markers in the EVs, including TSG101, syntenin 1, CD63, and CD9. In line with the in-vitro-based findings, treatment with ATM-derived exosomes from lean mice ameliorated dietinduced glucose intolerance and insulin resistance in obese mice, whereas administration of exosomes isolated from ATMs of obese mice promoted glucose intolerance and insulin resistance in lean recipients (13). These studies collectively support a critical role of ATM-derived exosomes in the regulation of neighboring adipocytes under physiological and pathological conditions.

\section{Adipose-Derived Stem Cells (ADSCs)}

ADSCs have emerged as a potential tool for regenerative therapy due to its multipotency in differentiating into different types of cells (58). Additionally, ADSCs are also a critical player in immune regulation, and have shown potential for treatment of inflammatory and autoimmune diseases, including colitis, autoimmune diabetes and arthritis, as well as to resolve obesity-induced inflammation and metabolic dysregulation by polarization of macrophages toward the anti-inflammatory M2 phenotypes (59-62). These beneficial effects may be attributed at least in part to the paracrine effects of EVs produced from ADSCs. Zhao et al. isolated ADSCs from mouse VAT and found that ADSC-derived EVs were approximately $100 \mathrm{~nm}$ in diameter and positive for the exosomal markers TGS101, CD9, CD63, HSP90, and ALIX, thus of exosomal origin (15). It has been shown in another study that human primary ADSCs also secreted $40-100 \mathrm{~nm}$ particles, which had the typical characteristics of exosomes (63). However, Katsuda et al. reported that human ADSC-derived exosomes had a peak size distribution of 150$200 \mathrm{~nm}$ which was larger than that reported by others. However, exosomal markers CD63 and HSP90 were present, suggesting that the size range of exosomes may differ among different cell types (64).

ADSC-derived exosomes isolated from patients with and without cancer show distinct miRNA profiles. Selective enrichment of certain miRNAs, including let-7-a-1, miR-21, and miR-1260b has been identified in ADSC-derived exosomes from cancer patients (65). Treatment of hepatocellular carcinoma cells with ADSC-exosomes containing miR-122 showed increased sensitivity to chemotherapies (66). Human ADSC-derived exosomes promoted migration of breast cancer cell line (63). ADSC-derived exosomes can be internalized into ATMs, and treatment of obese mice with ADSC-derived exosomes isolated from mouse VAT attenuated obesity and insulin resistance by inducing polarization of macrophages toward the M2 phenotypes through transactivation of arginase-1 by exosomecarried active STAT3, thus beiging of WAT (15). In addition to the undifferentiated ADSCs, EVs isolated from human ADSCs during white and beige adipogenic differentiation provided biochemical cues such as miRNAs to induce the differentiation of ADSCs into white and beige adipocytes, thereby promoting adipogenesis and adipose tissue remodeling, respectively (67).

\section{Endothelial Cells}

A recent study also identified adipose tissue endothelial cells as a source of ADEVs (68). These ADEVs are enriched with the exosomal markers CD9, CD63, TSG101, and ALIX. Production of EVs from adipose endothelial cells was increased under the fasted condition, mainly through the action of glucagon. As endothelial cells are located at the interface between the circulation and adipose tissue extracellular space, endothelial cell-derived ADEVs can take up proteins and lipids such as mitochondrial components and ceramides from the bloodstream, and subsequently release the components to the adjacent adipocytes through internalization (68). Notably, the changes of EV secretion from adipose endothelial cells in response to fasting and refeeding was absent in dietary or genetic obese mouse 
models, implicating the possible involvement of dysregulated adipose endothelial cell-derived EVs in the pathogenesis of obesity and its related metabolic diseases.

\section{MAJOR COMPONENTS OF ADEVs AND THEIR ROLES IN IMMUNE RESPONSES AND METABOLIC REGULATION}

EVs exert their biological functions by carrying various types of bioactive cargos including mRNAs, miRNAs, DNA, proteins and lipids to their target cells through phagocytosis or endocytosis $(55,69,70)$, which in turn mediate cell-cell communications. Additionally, since EVs have the same transmembrane proteins on their surface as the cell of origin, they can also act as the ligands directly binding and activating the surface receptor of target cells to initiate cellular signaling (71). Likewise, ADEVs modulate immune responses in local adipose tissues through cell-cell communication, and systemic insulin sensitivity, glucose and lipid metabolisms through their distal effects on other major metabolic organs such as liver, skeletal muscle, and brain (Figure 2). Such local and distal effects of ADEVs are attributed to their unique vesicular composition, which has been characterized in great details (Table 1).

\section{miRNA, mRNA and IncRNA}

The exosome-mediated cellular signaling is largely dependent on their composition of miRNAs, which are small non-coding RNA molecules that post-transcriptionally regulate gene expression by binding to the $3^{\prime}$-untranscribed region of target mRNAs, leading to mRNA degradation and repression of translation. miRNAs are critically involved in adipogenesis and regulation of adipose tissue functions (77). Recently, ADEVs have been found as an important source of circulating miRNAs in both mice and humans. It was evidenced by a significant reduction in exosomal miRNAs in serum of adipocyte-specific Dicer knockout (ADicerKO) mice, which have abrogated miRNA processing in adipocytes (12). DICER is a key enzyme that cleaves pre-miRNAs into mature miRNA, thus important for miRNA biogenesis (78). The same study also examined the circulating exosomal miRNA profiles in patients with congenital generalized lipodystrophy and patients with HIV-associated lipodystrophy who have general loss of adipose tissue and reduced expression of adipose Dicer respectively, and found that dominant miRNAs in exosomes were significantly downregulated in the serum of both patient cohorts, suggesting that circulating miRNAs in humans also originate mainly from ADEVs (12). Defects in ADEV-derived miRNA production resulted in reduced WAT, whitening of BAT, insulin resistance and dyslipidaemia in the ADicerKO mice, demonstrating the importance of adipose tissue-specific exosomal miRNAs in the physiological regulation of systemic energy metabolism. Transplantation of BAT, but not WAT from wild-type mice in ADicerKO mice improved glucose tolerance and insulin resistance in the recipient mice which was associated with reduced production and secretion of FGF21 from the liver (12). Further investigation revealed that mRNA expression of Fgf21 in hepatocytes was suppressed by ADEV-derived
miR-99b from BAT, suggesting that BAT-derived exosomal miRNAs mediate the adipose-liver crosstalk to modulate glucose homeostasis. However, both animal and clinical studies have shown beneficial effects of FGF21 in improving insulin sensitivity and alleviating hyperglycemia (79). Therefore, it is unlikely that the metabolic benefits of BAT-derived ADEVs are attributed to reduction in hepatic FGF21 expression. Further studies are needed to investigate the detailed molecular mechanism underlying the effects of BAT-derived exosomal miRNAs in the regulation of systemic glucose homeostasis.

ADEVs derived from different adipose depots appear to contain distinct miRNA composition. For example, miR-34a is selectively enriched in the exosomes from VAT, but not in SAT in both rodents and humans (11). Furthermore, high fat diet feeding leads to a progressive increase of miR$34 \mathrm{a}$ in the exosomes isolated from adipocytes in VAT in mice. The adipocyte-derived miR-34a is transported to the adjacent macrophages by exosomal delivery and drives the polarization of macrophages toward the pro-inflammatory M1 phenotypes by suppression of transcription factor Krüppellike factor 4 (KLF4), which is important in maintenance of M2 macrophage phenotypes. Conversely, adipocyte-selective ablation of miR-34a protects mice against obesity-induced adipose inflammation, systemic insulin resistance and NAFLD (11). Selective enrichment of miR-34a in VAT may explain why this adipose depot is more susceptible to inflammation and is more harmful to cardiometabolic health than SAT. In addition, miRNA-containing exosomes released from ATMs can modulate systemic insulin resistance. Administration of obese ATM-derived exosomes in lean mice impaired insulin sensitivity and glucose tolerance $(13,80)$. Uptake of ATM-exosomes can be detected in the liver, muscle and adipose tissues of mice. In-vitro experiments showed that exosomes derived from obese ATMs directly impaired insulin signaling in adipocytes, myocytes and hepatocytes (80). These effects were possibly attributed to obesity-induced changes in miRNA contents in the ATMexosomes, such as miR-155, which target the nuclear receptor PPAR $\gamma$ (13). Likewise, the distal effects of the exosomal miR27a released from adipocytes of obese mice on induction of insulin resistance in skeletal muscle were also attributed to its repression of PPAR $\gamma$ (73). Adipocytes also regulate lipid catabolism in skeletal muscle via exosomal miR-130b. miR130b has been shown to inhibit the expression of PPAR $\gamma$ coactivator $1 \alpha(\mathrm{PGC} 1 \alpha)$, which is important in lipid oxidative capacity and mitochondrial function (72). In addition to the aforementioned roles of miR-155 on adipocytes, hepatocytes and myocytes, exosomal miR-155 derived from ATMs of obese mice also exerts profound regulation on pancreatic $\beta$ cells, leading to impaired insulin secretion and increased $\beta$ cell proliferation by repressing the expression of $\mathrm{v}$-maf musculoaponeurotic fibrosarcoma oncogene family protein B (MAFB) (74).

There is emerging evidence showing that environmental changes can alter the composition of adipose-derived exosomal miRNA, which in turn participates in adaptive responses to metabolic stresses. In high-attitude population, hypoxia and cold temperature causes downregulation of exosomal miR210/92a from WAT, thereby increasing the thermogenic activity 


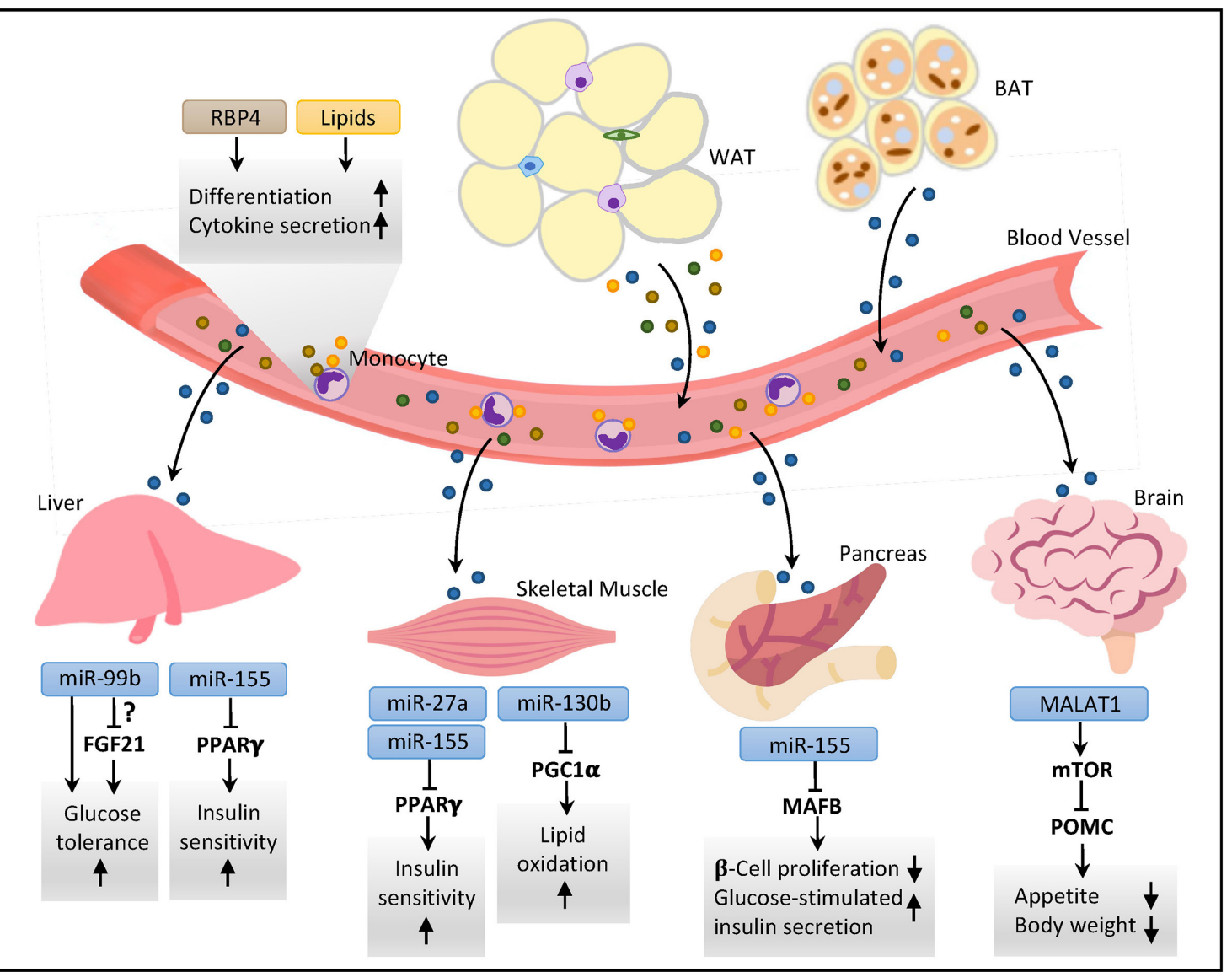

FIGURE 2 | ADEVs-mediated interorgan crosstalk in metabolic regulation. Both white and brown adipose tissues secrete EVs containing various types of vesicular components into the circulation. The ADEVs can act as endocrine factors affecting metabolic profiles in distal organs by sending bioactive vesicular molecules. In the liver, exosomal miRNAs modulate glucose tolerance and insulin sensitivity through modulation of peroxisome proliferator activated receptor gamma (PPAR $\gamma$ ) and perhaps fibroblast growth factor 21 (FGF21). In skeletal muscle, miRNAs regulate insulin sensitivity and lipid oxidative capacity through PPAR $\gamma$ and PPAR $\gamma$ coactivator $1 \alpha(P G C 1 \alpha)$, respectively. In pancreas, ADEV-derived miRNAs modulate $\beta$-cell mass and insulin secretion. In brain, ADEVs-derived long non-coding RNA metastasis-associated lung adenocarcinoma transcript-1 (MALAT1) regulates mTOR signaling in hypothalamic pro-opiomelanocortin (POMC) neurons to control appetite and body weight. In the bloodstream, exosomal proteins and lipids affect the differentiation and immunomodulatory responses of monocytes. RBP4, retinol binding protein 4; WAT, white adipose tissue; BAT, brown adipose tissue; MAFB, v-maf musculoaponeurotic fibrosarcoma oncogene family protein B.

of BAT possibly by upregulation of FGFR1 (81). ADEVs may participate in the regulation of the inflammasome activation. EVs derived from both ADSCs and epidural fat-mesenchymal stem cells inhibit Nod-like receptor pyrin domain-containing three (NLRP3) inflammasome activation $(82,83)$. MiR-223, possibly of ADEV origin, is reduced in blood from patients with T2D and obesity (84). This reduction in miR-223 is believed to contribute to the increased adipose tissue inflammation in obesity as miR223 can inhibit inflammation by targeting NLRP3, which is a key component of the inflammasome (85). However, it is currently unclear how adipose tissues sense the environmental and nutritional changes to alter the vesicular composition of miRNAs under different pathophysiological conditions.

In addition to miRNAs, mRNAs have been found to be present in EVs. Valadi and colleagues provided the first evidence demonstrating that EVs secreted from mast cells contained substantial amount of mRNAs, which were functional as they can be transferred to other cells and translated into new proteins in the recipient cells (70). Subsequent analysis of EVs derived from adipocytes differentiated from mouse 3T3-L1 cell line revealed that ADEVs also contained mRNAs encoding genes involved in metabolic and inflammatory processes (86). In contrast, exosomal mRNAs were not detected in VAT or SAT from either lean or obese human subjects (87). The discrepancy may be resulted from the difference in cellular sources of exosomes and possible difference in miRNA species that might mediate intrinsic degradation of mRNAs in ADEVs from human subjects.

Long non-coding RNAs (lncRNAs) have been emerged as critical regulators to control the development and functions of various metabolic tissues. For example, brown adipose tissuespecific lncRNA 1 (lnc-BATE1) was induced during brown adipocyte differentiation and enriched in BAT compared to WAT in mice. siRNA-mediated knockdown of lnc-BATE1 impaired differentiation of brown adipocytes in vitro (88). Liver-specific 
TABLE 1 | Summary of major ADEV cargos and their functions in immune responses and metabolic regulation.

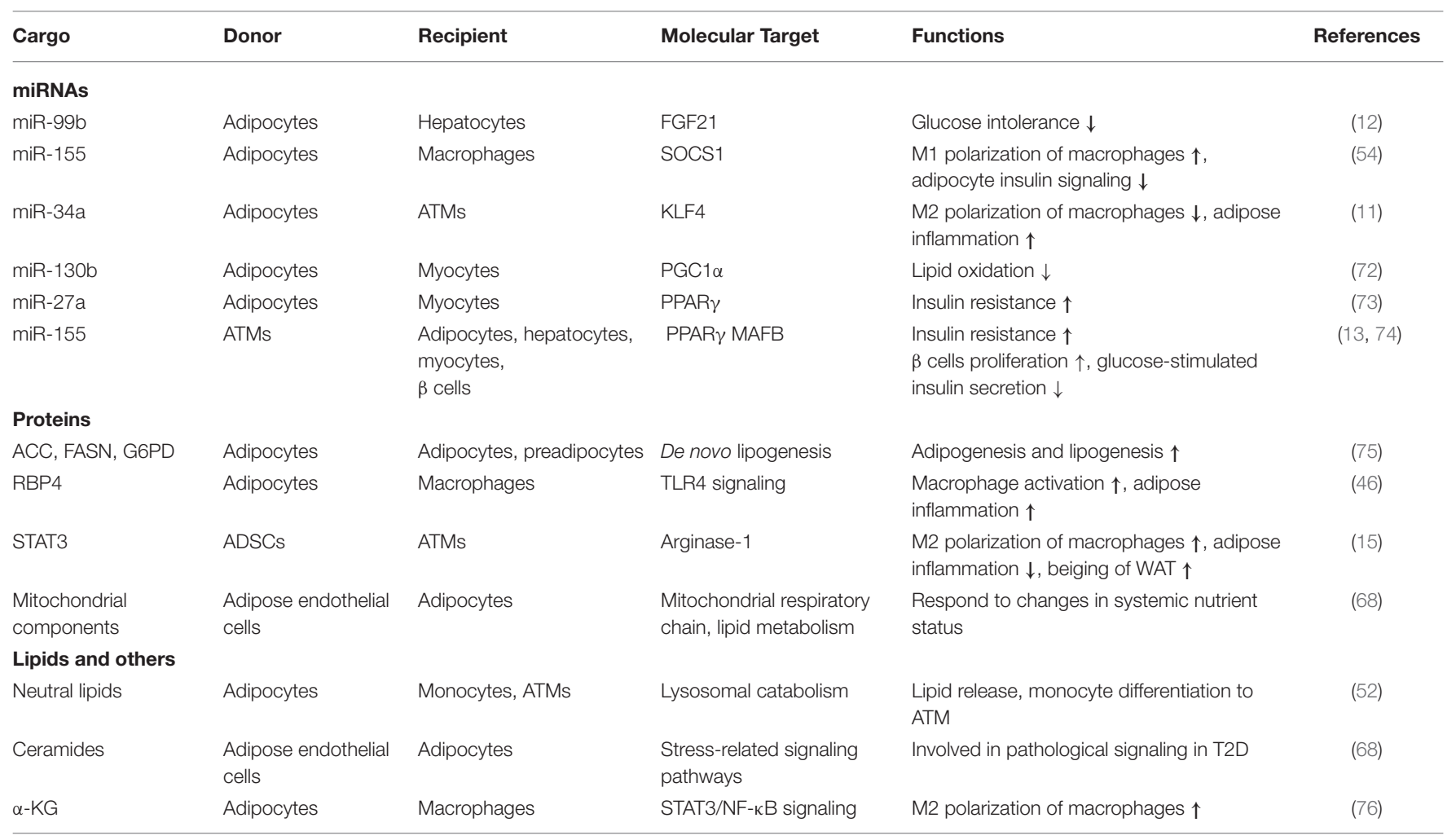

FGF21, fibroblast growth factor 21; SOCS1, suppressor of cytokine signaling 1; ATMS, adipose tissue-resident macrophages; KLF4, krüppel-like factor 4; PPARy, peroxisome proliferator activated receptor gamma; PGC1 $\alpha$, PPAR $\gamma$ coactivator $1 \alpha$; MAFB, v-maf musculoaponeurotic fibrosarcoma oncogene family protein $B ; A C C$, acetyl-CoA carboxylase; FASN, fatty acid synthase; G6PD, glucose-6-phosphate dehydrogenase; RBP4, retinol binding protein 4; TLR4, toll-like receptor 4; STAT3, signal transducer and activator of transcription 3; ADSCs, adipose-derived stem cells; WAT, white adipose tissue; T2D, type 2 diabetes; $\alpha-K G$, $\alpha$-ketoglutarate; NF-KB, nuclear factor kappa B.

triglyceride regulator lncRNA (lnc-LSTR) was identified as an important regulator of hepatic lipid metabolism. Knockdown of lnc-LSTR in mice lowered serum triglyceride levels by induction of apolipoprotein C2 (ApoC2), which promotes lipoprotein lipase-mediated hydrolysis of triglyceride-rich lipoproteins (89). Recently, it has been reported that lncRNAs are transferred by ADEVs to mediate the interconnection between adipose tissue and the central nervous system. In particular, adipocyte-derived exosomal metastasis-associated lung adenocarcinoma transcript1 lncRNA (lnc-MALAT1), which is elevated in obese mice, has been shown to target hypothalamic pro-opiomelanocortin (POMC) neurons to upregulate mTOR and thereby downregulate POMC expression, resulting in increased appetite and weight gain in lean mice (90).

\section{Proteins}

The nature of proteins released from adipocyte-derived exosomes has been characterized by proteomic profiling of exosomes produced by human primary adipocytes in comparison with the overall secretome of the same cells (69). This analysis identified 884 proteins, called as exoadipokines. Among them, 212 proteins commonly found in both secretome of human adipose tissues and exosomes are mainly involved in inflammation and fibrosis, whereas 672 proteins specific for exosomes which are assigned to signaling pathways and membrane-mediated process (69).
Notably, exosomes were found to be enriched in proteins without classical signaling peptides that direct proteins to the traditional secretory pathway, suggesting a significant contribution of exosomes to the overall human adipokinome (69). Notably, different proteomic profiles in adipocyte-derived EVs have been observed between obese diabetic rats and obese non-diabetic counterparts (91). Exosomes derived from obese diabetic mice are enriched in proteins and enzymes involved in lipolysis and glycerol export, which may explain ectopic lipid accumulation in major metabolic organs and hence systemic insulin resistance (91). Intriguingly, hypoxia, which is present in obese adipose tissue, has also been shown to alter proteomic composition of ADEVs in vitro (75). In particular, enzymes involved in de novo lipogenesis such as acetyl-CoA carboxylase (ACC), fatty acid synthase (FASN) and glucose-6-phosphate dehydrogenase (G6PD) were selectively enriched in exosomes derived from adipocytes under the hypoxic condition, which may increase lipid accumulation in recipient adipocytes and preadipocytes.

Exosomal proteins in ADEVs are functionally involved in the paracrine crosstalk between adipocytes and macrophages in adipose tissue. Adiponectin-positive EVs derived from adipocytes can promote differentiation of monocytes into ATMs, which are associated with the production of immunomodulatory proteins such as $\mathrm{TNF} \alpha$, macrophage-colony-stimulating factor (MCSF) and retinol binding protein 4 (RBP4) (47). The 
functional relevance of exosomal protein RBP4 in monocyte differentiation has been substantiated in another independent study showing that ADEVs from ob/ob mice contained a higher level of RBP4, and exosomal RBP4 induced macrophage activation and production of pro-inflammatory cytokines in vitro (46). Additionally, exosomes from ADSCs contain active STAT3, which can be transported to macrophages to induce polarization of macrophages toward the anti-inflammatory M2 phenotypes through transcriptional activation of arginase-1 (15). Treatment of obese mice with the ADSC-derived exosomes alleviated diet-induced insulin resistance and glucose intolerance by reducing adipose inflammation and enhancing beiging of WAT (15). These studies collectively support the immunomodulatory effects of exosomal proteins, in reminiscence of classical adipokines and chemokines, in the interconnection between obesity and inflammation. However, loss-of-function studies are warranted to confirm the requirement of individual exosomal proteins in the regulation of adipose immune responses and insulin sensitivity.

\section{Lipids and Other Cargos}

In addition to aforementioned miRNAs, mRNAs and proteins, lipids and other cargos also act as the signaling molecules conferring the effects of ADEVs. A recent study identified lipid-filled exosomes released from adipocytes, and these lipidenriched exosomes play an important role in transporting lipids from adipocytes to macrophages (52). Furthermore, these lipidfilled exosomes are also sufficient to induce differentiation of bone marrow-derived monocytes into ATM-like macrophages in vitro (52). The number of the lipid-filled exosomes secreted from adipocytes was more than doubled in obese mice relative to the lean mice which might be an additional mechanism for obesity-associated adipose inflammation. This study also proposed these lipid-filled exosomes as a new pathway of lipid release from adipocytes independent of the canonical lipolysis. However, little is known on how different types of bioactive lipids are selectively enriched in ADEVs and exert their local and/or distal effects on immunological and metabolic regulation. Adipocytes are also found to produce exosomes containing $\alpha$-ketoglutarate. Melatonin, a hormone released from the pineal gland with anti-inflammatory activities, promoted the secretion of exosomes containing $\alpha$-ketoglutarate from adipocytes, whereas uptake of exosomal $\alpha$-ketoglutarate by macrophages facilitated the polarization toward the antiinflammatory M2 phenotype and thus alleviated adipose inflammation in obesity (76).

\section{CLINICAL IMPLICATIONS OF ADEVs IN METABOLIC DISEASES}

Measurement of ADEVs in adipose tissue is minimally invasive and can be potentially used as an alternative approach to evaluate metabolic health. Changes in circulating EVs have been associated with various metabolic diseases, including obesity, T2D and NAFLD, making them attractive biomarkers for diagnosis and risk prediction of these diseases (92). However, the extent to which circulating EVs are contributed by
ADEVs is currently unclear. Several adipose markers, including adiponectin, FABP4 and perilipin A have been used to identify EVs released from adipose tissues $(50,51)$. The level of circulating EVs positive for perilipin $\mathrm{A}$ and thus of adipocyte origin was dramatically increased in both mice with diet-induced obesity and obese human patients (50). Furthermore, in obese humans, the circulating level of the EVs enriched with perilipin A was positively correlated with plasma insulin level and homeostatic model assessment of insulin resistance (HOMA-IR), supporting potential use of perilipin A-positive EVs as the biomarker of insulin resistance (50).

Metabolic status can be also reflected by changes in vesicular miRNAs in ADEVs. By using FABP4 as a marker to identify ADEVs from the circulation, Hubal et al. found that the miRNA content of circulating ADEVs targeting various genes in the canonical insulin receptor-mediated signaling pathway was significantly altered 1 year-after gastric bypass bariatric surgery, and the changes were closely associated with improvements in insulin sensitivity, suggesting that FABP4-positive ADEVs might be useful to monitor the response of obese patients to the intervention with bariatric surgery (51). However, as FABP4 is also expressed in several types of immune cells and endothelial cells, ADEVs may only account for a proportion of the total FABP4 positive EVs. In addition, the circulating level of exosomal miR-92a possibly of BAT origin was found to be negatively associated with BAT activity as measured by ${ }^{18} \mathrm{~F}$-fluorodeoxyglucose $\mathrm{PET} / \mathrm{CT}$ in two human cohorts, and thus may represent a potential biomarker for monitoring BAT activity in humans, which is much more cost-effective than PET/CT-based approaches (53).

ADEVs from different adipose depots are differentially associated with metabolic health and disease. The number of EVs derived from omental adipose tissue, but not SAT, correlated positively with HOMA-IR in overweight patients (47). It has also been reported that the abundance of EVs in VAT was positively correlated with serum levels of alanine aminotransferase (ALT) and aspartate aminotransferase (AST), which are the wellestablished markers for liver injury, whereas the number of EVs in SAT was inversely associated with waist circumference and metabolic syndrome (93). These clinical observations suggest that differential effects of SAT and VAT on metabolic health may be related to the distinct amount and/or composition of EVs from these two adipose depots.

\section{THERAPEUTIC POTENTIAL OF ADEVs FOR METABOLIC DISEASES}

Owing to the easy accessibility from the bloodstream, the ability to transport the bioactive cargos and surmount biological barriers, the possibilities to modify the content with bioengineering, and target specificity, EVs have been emerged as a cell-free therapy for treatment of various diseases (94). In particular, EVs from mesenchymal stem cells (MSCs) could fully mimic the immunomodulatory and regenerative functions of parental MSCs, and have therefore been exploited as potential therapeutic agents for various inflammatory diseases 
and regenerative medicine targeting lung, liver, bone, kidney, brain and heart (95), and a large number of clinical trials on MSC-derived EVs have been publicly registered in recent years.

Although ADSCs have been shown to possess promising therapeutic efficacy for Crohn's disease, idiopathic pulmonary fibrosis and chronic kidney diseases (NCT03939741) in various clinical studies, the therapeutic application of ADSC-derived EVs are still at the early stage $(96,97)$. Nevertheless, there is a growing number of preclinical studies suggesting that ADEVs have the great therapeutic potential for obesity-related metabolic diseases. Treatment of obese mice with ADSCderived exosomes obtained from lean mice caused a significant reduction of adipose inflammation and beiging of WAT, thereby leading to obvious metabolic improvements, including weight loss, alleviation of insulin resistance and hepatic steatosis (15). Administration of exosomes isolated from BAT or serum of wild-type mice significantly improved insulin sensitivity and normalized serum lipids in ADicerKO mice (12). Likewise, treatment of mice with EVs isolated from human ADSCs during adipogenic differentiation to beige adipocytes attenuated diet-induced obesity and hepatic steatosis (67). By contrast, infusion of mice with EVs isolated from human ADSCs during adipogenic induction to white adipocytes promoted adipogenesis and expansion of WAT, suggesting the therapeutic potential for lipodystrophy, a disorder associated with reduced number of circulating exosomes (12).

In addition to use endogenous $\mathrm{ADEVs}$ as a therapeutic agent, bioengineering ADEVs by modifying the bioactive cargos may represent another viable approach to develop effective treatment for obesity-related metabolic complications. An example is to deplete those miRNAs causally involved in metabolic inflammation and insulin resistance. In this connection, genetic ablation of exosomal miR-34a, which is highly enriched in exosomes secreted from VAT and contributes to adipose inflammation by inducing M1 macrophage polarization, has been shown to reverse obesity-induced insulin resistance, glucose intolerance and fatty liver in mice (11). Similarly, treatment of dietary obese mice with antisense RNA for miR$34 \mathrm{a}$ restores hepatic $\beta$-klotho expression and FGF19 signaling, leading to attenuation of fatty liver disease (98). It is also possible to exogenously load ADEVs with transcriptional factors participating in M2 macrophage polarization (15), thereby reducing obesity-related metabolic diseases.

\section{CONCLUSIONS AND FUTURE PERSPECTIVES}

Emerging evidence from both in-vitro and in-vivo studies support the role of ADEVs as important players mediating

\section{REFERENCES}

1. Malik VS, Willett WC, Hu FB. Global obesity: trends, risk factors and policy implications. Nat Rev Endocrinol. (2013) 9:13-27. doi: 10.1038 /nrendo.2012.199 cell-cell communication within adipose tissues as well as interorgan crosstalk between adipose tissue and other distal organs, thus participating in the regulation of local immune responses, tissue remodeling, systemic insulin sensitivity, and energy homeostasis (Figures 1, 2). Aberrant production and/or function of ADEVs are implicated in the pathogenesis of obesity and its related metabolic complications. ADEVs are heterogeneous in terms of size, composition and origin, with ADEVs derived from different adipose depots exhibiting distinct or even opposite functions. However, we are still in the early stage in understanding biogenesis, regulation and pathophysiological functions of ADEVs, and there are many important questions which remain to be addressed: How is the cargo composition of ADEVs regulated? How does obesity cause dysregulation in the number and cargo composition of ADEVs? What determines the target specificity of ADEVs? How do different cargos affect the functions of target cells? Furthermore, the clinical investigation of ADEVs as diagnostic biomarkers and therapeutics for metabolic diseases are constrained by several technical difficulties: First, there is no well-established, definitive marker(s) for ADEVs, and it is therefore difficult to dissect the contribution of ADEVs to circulating EVs, and to precisely measure the changes of circulating ADEVs in different metabolic diseases. Second, it is difficult to isolate ADEVs with high purity using current experimental approaches, and contamination with other particles such as lipoproteins remains a major concern. Moreover, due to the heterogeneity of ADEVs, a mixture of EV populations with different cargos exists in the same cell, causing the difficulties in obtaining a subtype with a specific set of cargos for functional characterization. Further technological advances in molecular and functional characterization of ADEVs will help to enhance our knowledge in metabolic regulation and to facilitate the development of novel therapeutics for treatment of obesity and its related metabolic complications.

\section{AUTHOR CONTRIBUTIONS}

$\mathrm{ZH}$ designed the review, collected references, prepared the table and figures, and wrote the manuscript. AX conceptualized the idea, provided critical suggestions and edited the manuscript. All authors contributed to the article and approved the submitted version.

\section{FUNDING}

This work was supported by The National Key Research and Development Program of China (2016YFC1305003), Hong Kong Research Grants Council/Area of Excellence (AoE/M/707-18), Collaborative Research Fund (C7037-17W).
2. Stefan N, Birkenfeld AL, Schulze MB, Ludwig DS. Obesity and impaired metabolic health in patients with COVID-19. Nat Rev Endocrinol. (2020) 16:341-2. doi: 10.1038/s41574-020-0364-6

3. Fasshauer M, Blüher M. Adipokines in health and disease. Trends Pharmacol Sci. (2015) 36:461-70. doi: 10.1016/j.tips.2015.04.014 
4. Unamuno X, Gómez-Ambrosi J, Rodríguez A, Becerril S, Frühbeck G, Catalán $\mathrm{V}$. Adipokine dysregulation and adipose tissue inflammation in human obesity. Eur J Clin Invest. (2018) 48:e12997. doi: 10.1111/eci.12997

5. Rosen ED, Spiegelman BM. What we talk about when we talk about fat. Cell. (2014) 156:20-44. doi: 10.1016/j.cell.2013.12.012

6. Hotamisligil GS, Arner P, Caro JF, Atkinson RL, Spiegelman BM. Increased adipose tissue expression of tumor necrosis factor-alpha in human obesity and insulin resistance. J Clin Invest. (1995) 95:2409-15. doi: 10.1172/JCI117936

7. Hotamisligil GS, Shargill NS, Spiegelman BM. Adipose expression of tumor necrosis factor-alpha: direct role in obesity-linked insulin resistance. Science. (1993) 259:87-91. doi: 10.1126/science.7678183

8. Arkan MC, Hevener AL, Greten FR, Maeda S, Li Z-W, Long JM, et al. IKK$\beta$ links inflammation to obesity-induced insulin resistance. Nat Med. (2005) 11:191-8. doi: $10.1038 / \mathrm{nm} 1185$

9. Hirosumi J, Tuncman G, Chang L, Görgün CZ, Uysal KT, Maeda K, et al. A central role for JNK in obesity and insulin resistance. Nature. (2002) 420:333-6. doi: 10.1038/nature01137

10. Zhang X, Xu A, Chung SK, Cresser JH, Sweeney G, Wong RL, et al. Selective inactivation of c-Jun $\mathrm{NH}$-terminal kinase in adipose tissue protects against diet-induced obesity and improves insulin sensitivity in both liver and skeletal muscle in mice. Diabetes. (2011) 60:486-95. doi: 10.2337/db10-0650

11. Pan Y, Hui X, Hoo RLC, Ye D, Chan CYC, Feng T, et al. Adipocyte-secreted exosomal microRNA-34a inhibits M2 macrophage polarization to promote obesity-induced adipose inflammation. J Clin Invest. (2019) 129:834-49. doi: 10.1172/JCI123069

12. Thomou T, Mori MA, Dreyfuss JM, Konishi M, Sakaguchi M, Wolfrum C, et al. Adipose-derived circulating miRNAs regulate gene expression in other tissues. Nature. (2017) 542:450-5. doi: 10.1038/nature21365

13. Ying W, Riopel M, Bandyopadhyay G, Dong Y, Birmingham A, Seo JB, et al. Adipose tissue macrophage-derived exosomal miRNAs can modulate in vivo and in vitro insulin sensitivity. Cell. (2017) 171:372-84.e312. doi: 10.1016/j.cell.2017.08.035

14. Zhang Y, Shi L, Mei H, Zhang J, Zhu Y, Han X, et al. Inflamed macrophage microvesicles induce insulin resistance in human adipocytes. Nutr Metab. (2015) 12:1-14. doi: 10.1186/s12986-015-0016-3

15. Zhao H, Shang Q, Pan Z, Bai Y, Li Z, Zhang H, et al. Exosomes from adiposederived stem cells attenuate adipose inflammation and obesity through polarizing M2 macrophages and beiging in white adipose tissue. Diabetes. (2018) 67:235-47. doi: 10.2337/db17-0356

16. Booth A, Magnuson A, Foster M. Detrimental and protective fat: body fat distribution and its relation to metabolic disease. Horm Mol Biol Clin Investig. (2014) 17:13-27. doi: 10.1515/hmbci-2014-0009

17. Lee M-J, Wu Y, Fried SK. Adipose tissue heterogeneity: implication of depot differences in adipose tissue for obesity complications. Mol Aspects Med. (2013) 34:1-11. doi: 10.1016/j.mam.2012.10.001

18. Ibrahim MM. Subcutaneous and visceral adipose tissue: structural and functional differences. Obes Rev. (2010) 11:11-8. doi: 10.1111/j.1467-789X.2009.00623.x

19. Saltiel AR, Kahn CR. Insulin signalling and the regulation of glucose and lipid metabolism. Nature. (2001) 414:799-806. doi: 10.1038/414799a

20. Fedorenko A, Lishko PV, Kirichok Y. Mechanism of fatty-acid-dependent UCP1 uncoupling in brown fat mitochondria. Cell. (2012) 151:400-13. doi: 10.1016/j.cell.2012.09.010

21. Nedergaard J, Golozoubova V, Matthias A, Asadi A, Jacobsson A, Cannon B. UCP1: the only protein able to mediate adaptive non-shivering thermogenesis and metabolic inefficiency. Biochim Biophys Acta Bioenerget. (2001) 1504:82-106. doi: 10.1016/S0005-2728(00)00247-4

22. Lee Y-H, Petkova AP, Mottillo EP, Granneman JG. In vivo identification of bipotential adipocyte progenitors recruited by $\beta 3$-adrenoceptor activation and high-fat feeding. Cell Metab. (2012) 15:480-91. doi: 10.1016/j.cmet.2012.03.009

23. Wu J, Boström P, Sparks LM, Ye L, Choi JH, Giang A-H, et al. Beige adipocytes are a distinct type of thermogenic fat cell in mouse and human. Cell. (2012) 150:366-76. doi: 10.1016/j.cell.2012.05.016

24. Cypess AM, Lehman S, Williams G, Tal I, Rodman D, Goldfine AB, et al. Identification and importance of brown adipose tissue in adult humans. $N$ Engl J Med. (2009) 360:1509-17. doi: 10.1056/NEJMoa0810780
25. van Marken Lichtenbelt WD, Vanhommerig JW, Smulders NM, Drossaerts JM, Kemerink GJ, Bouvy ND, et al. Cold-activated brown adipose tissue in healthy men. N Engl J Med. (2009) 360:1500-8. doi: 10.1056/NEJMoa0808718

26. Virtanen KA, Lidell ME, Orava J, Heglind M, Westergren R, Niemi T, et al. Functional brown adipose tissue in healthy adults. N Engl J Med. (2009) 360:1518-25. doi: 10.1056/NEJMoa0808949

27. Ouellet V, Routhier-Labadie A, Bellemare W, Lakhal-Chaieb L, Turcotte E, Carpentier AC, et al. Outdoor temperature, age, sex, body mass index, and diabetic status determine the prevalence, mass, and glucose-uptake activity of 18F-FDG-detected BAT in humans. J Clin Endocrinol Metab. (2011) 96:192-9. doi: 10.1210/jc.2010-0989

28. Hollenberg C, Vost A. Regulation of DNA synthesis in fat cells and stromal elements from rat adipose tissue. J Clin Invest. (1968) 47:2485-98. doi: 10.1172/JCI105930

29. Panina YA, Yakimov AS, Komleva YK, Morgun AV, Lopatina OL, Malinovskaya NA, et al. Plasticity of adipose tissue-derived stem cells and regulation of angiogenesis. Front Physiol. (2018) 9:1656. doi: $10.3389 /$ fphys. 2018.01656

30. Cao Y. Adipose tissue angiogenesis as a therapeutic target for obesity and metabolic diseases. Nat Rev Drug Discov. (2010) 9:107-15. doi: $10.1038 / \mathrm{nrd} 3055$

31. Deshmukh AS, Peijs L, Beaudry JL, Jespersen NZ, Nielsen CH, Ma T, et al. Proteomics-based comparative mapping of the secretomes of human brown and white adipocytes reveals EPDR1 as a novel batokine. Cell Metab. (2019) 30:963-75.e967. doi: 10.1016/j.cmet.2019.10.001

32. Mahlakõiv T, Flamar A-L, Johnston L, Moriyama S, Putzel G, Bryce $\mathrm{P}$, et al. Stromal cells maintain immune cell homeostasis in adipose tissue via production of interleukin-33. Sci Immunol. (2019) 4:eaax0416. doi: 10.1126/sciimmunol.aax0416

33. Sun C, Berry WL, Olson LE. PDGFR $\alpha$ controls the balance of stromal and adipogenic cells during adipose tissue organogenesis. Development. (2017) 144:83-94. doi: 10.1242/dev.135962

34. Weisberg SP, McCann D, Desai M, Rosenbaum M, Leibel RL, Ferrante AW. Obesity is associated with macrophage accumulation in adipose tissue. J Clin Invest. (2003) 112:1796-808. doi: 10.1172/JCI200319246

35. Xu H, Barnes GT, Yang Q, Tan G, Yang D, Chou CJ, et al. Chronic inflammation in fat plays a crucial role in the development of obesity-related insulin resistance. J Clin Invest. (2003) 112:1821-30. doi: 10.1172/JCI200319451

36. Schipper HS, Prakken B, Kalkhoven E, Boes M. Adipose tissue-resident immune cells: key players in immunometabolism. Trends Endocrinol Metab. (2012) 23:407-15. doi: 10.1016/j.tem.2012.05.011

37. Cildir G, Akincilar SC, Tergaonkar V. Chronic adipose tissue inflammation: all immune cells on the stage. Trends Mol Med. (2013) 19:487-500. doi: 10.1016/j.molmed.2013.05.001

38. Tilg H, Moschen AR. Adipocytokines: mediators linking adipose tissue, inflammation and immunity. Nat Rev Immunol. (2006) 6:772-83. doi: 10.1038/nri1937

39. Nakamura K, Fuster JJ, Walsh K. Adipokines: a link between obesity and cardiovascular disease. J Cardiol. (2014) 63:250-9. doi: 10.1016/j.jjcc.2013.11.006

40. Hassan M, Latif N, Yacoub M. Adipose tissue: friend or foe? Nat Rev Cardiol. (2012) 9:689. doi: 10.1038/nrcardio.2012.148

41. Lumeng CN, Bodzin JL, Saltiel AR. Obesity induces a phenotypic switch in adipose tissue macrophage polarization. J Clin Invest. (2007) 117:175-84. doi: 10.1172/JCI29881

42. Shi H, Kokoeva MV, Inouye K, Tzameli I, Yin H, Flier JS. TLR4 links innate immunity and fatty acid-induced insulin resistance. J Clin Invest. (2006) 116:3015-25. doi: 10.1172/JCI28898

43. Sica A, Mantovani A. Macrophage plasticity and polarization: in vivo veritas. J Clin Invest. (2012) 122:787-95. doi: 10.1172/JCI59643

44. Van der Pol E, Böing AN, Harrison P, Sturk A, Nieuwland R. Classification, functions, and clinical relevance of extracellular vesicles. Pharmacol Rev. (2012) 64:676-705. doi: 10.1124/pr.112.005983

45. Colombo M, Raposo G, Théry C. Biogenesis, secretion, and intercellular interactions of exosomes and other extracellular vesicles. Annu Rev Cell Dev Biol. (2014) 30:255-89. doi: 10.1146/annurev-cellbio-101512-122326 
46. Deng Z-b, Poliakov A, Hardy RW, Clements R, Liu C, Liu Y, et al. Adipose tissue exosome-like vesicles mediate activation of macrophage-induced insulin resistance. Diabetes. (2009) 58:2498-505. doi: 10.2337/db09-0216

47. Kranendonk ME, Visseren FL, van Balkom BW, Nolte-'t Hoen EN, van Herwaarden JA, de Jager W, et al. Human adipocyte extracellular vesicles in reciprocal signaling between adipocytes and macrophages. Obesity. (2014) 22:1296-308. doi: 10.1002/oby.20679

48. Phoonsawat W, Aoki-Yoshida A, Tsuruta T, Sonoyama K. Adiponectin is partially associated with exosomes in mouse serum. Biochem Biophys Res Commun. (2014) 448:1-266. doi: 10.1016/j.bbrc.2014.04.114

49. Aoki N, Jin-No S, Nakagawa Y, Asai N, Arakawa E, Tamura N, et al. Identification and characterization of microvesicles secreted by 3T3-L1 adipocytes: redox-and hormone-dependent induction of milk fat globuleepidermal growth factor 8-associated microvesicles. Endocrinology. (2007) 148:3850-62. doi: 10.1210/en.2006-1479

50. Eguchi A, Lazic M, Armando AM, Phillips SA, Katebian R, Maraka $\mathrm{S}$, et al. Circulating adipocyte-derived extracellular vesicles are novel markers of metabolic stress. J Mol Med. (2016) 94:1241-53. doi: 10.1007/s00109-016-1446-8

51. Hubal MJ, Nadler EP, Ferrante SC, Barberio MD, Suh JH, Wang J, et al. Circulating adipocyte-derived exosomal MicroRNAs associated with decreased insulin resistance after gastric bypass. Obesity. (2017) 25:102-10. doi: $10.1002 /$ oby.21709

52. Flaherty SE, Grijalva A, Xu X, Ables E, Nomani A, Ferrante AW. A lipaseindependent pathway of lipid release and immune modulation by adipocytes. Science. (2019) 363:989-93. doi: 10.1126/science.aaw2586

53. Chen Y, Buyel JJ, Hanssen MJ, Siegel F, Pan R, Naumann J, et al. Exosomal microRNA miR-92a concentration in serum reflects human brown fat activity. Nat Commun. (2016) 7:1-9. doi: 10.1038/ncomms11420

54. Zhang Y, Mei H, Chang X, Chen F, Zhu Y, Han X. Adipocyte-derived microvesicles from obese mice induce M1 macrophage phenotype through secreted miR-155. J Mol Cell Biol. (2016) 8:505-17. doi: 10.1093/jmcb/mjw040

55. Clement E, Lazar I, Attan,é C, Carri,é L, Dauvillier S, Ducoux-Petit M, et al. Adipocyte extracellular vesicles carry enzymes and fatty acids that stimulate mitochondrial metabolism and remodeling in tumor cells. EMBO J. (2020) 39:e102525. doi: 10.15252/embj.2019102525

56. Lazar I, Clement E, Dauvillier S, Milhas D, Ducoux-Petit M, LeGonidec S, et al. Adipocyte exosomes promote melanoma aggressiveness through fatty acid oxidation: a novel mechanism linking obesity and cancer. Cancer Res. (2016) 76:4051-7. doi: 10.1158/0008-5472.CAN-16-0651

57. De Silva N, Samblas M, Martínez JA, Milagro FI. Effects of exosomes from LPS-activated macrophages on adipocyte gene expression, differentiation, and insulin-dependent glucose uptake. J Physiol Biochem. (2018) 74:559-68. doi: 10.1007/s13105-018-0622-4

58. Mizuno H, Tobita M, Orbay H, Uysal AC, Lu F. Adipose-derived stem cells as a novel tool for future regenerative medicine. Stem Cells Cancer Stem Cells. (2014) 12:165-74. doi: 10.1007/978-94-017-8032-2_15

59. Bassi ÊJ, Moraes-Vieira PM, Moreira,-S.á CS, Almeida DC, Vieira LM, Cunha CS, et al. Immune regulatory properties of allogeneic adipose-derived mesenchymal stem cells in the treatment of experimental autoimmune diabetes. Diabetes. (2012) 61:2534-45. doi: 10.2337/db11-0844

60. González MA, Gonzalez-Rey E, Rico L, Büscher D, Delgado M. Treatment of experimental arthritis by inducing immune tolerance with human adipose-derived mesenchymal stem cells. Arthritis Rheum. (2009) 60:1006-19. doi: 10.1002/art.24405

61. González MA, Gonzalez-Rey E, Rico L, Büscher D, Delgado M. Adiposederived mesenchymal stem cells alleviate experimental colitis by inhibiting inflammatory and autoimmune responses. Gastroenterology. (2009) 136:97889. doi: 10.1053/j.gastro.2008.11.041

62. Shang Q, Bai Y, Wang G, Song Q, Guo C, Zhang L, et al. Delivery of adipose-derived stem cells attenuates adipose tissue inflammation and insulin resistance in obese mice through remodeling macrophage phenotypes. Stem Cells Develop. (2015) 24:2052-64. doi: 10.1089/scd.2014.0557

63. Lin R, Wang S, Zhao RC. Exosomes from human adipose-derived mesenchymal stem cells promote migration through Wnt signaling pathway in a breast cancer cell model. Mol Cell Biochem. (2013) 383:13-20. doi: $10.1007 / \mathrm{s} 11010-013-1746-\mathrm{Z}$
64. Katsuda T, Tsuchiya R, Kosaka N, Yoshioka Y, Takagaki K, Oki K, et al. Human adipose tissue-derived mesenchymal stem cells secrete functional neprilysin-bound exosomes. Sci Rep. (2013) 3:1197. doi: 10.1038/srep01197

65. García-Contreras M, Vera-Donoso CD, Hernandez-Andreu JM, GarcíaVerdugo JM, Oltra E. Therapeutic potential of human adipose-derived stem cells (ADSCs) from cancer patients: a pilot study. PLoS ONE. (2014) 9:e113288. doi: 10.1371/journal.pone.0113288

66. Lou G, Song X, Yang F, Wu S, Wang J, Chen Z, et al. Exosomes derived from miR-122-modified adipose tissue-derived MSCs increase chemosensitivity of hepatocellular carcinoma. J Hematol Oncol. (2015) 8:1-11. doi: 10.1186/s13045-015-0220-7

67. Jung YJ, Kim HK, Cho Y, Choi JS, Woo CH, Lee KS, et al. Cell reprogramming using extracellular vesicles from differentiating stem cells into white/beige adipocytes. Sci Advanc. (2020) 6:eaay6721. doi: 10.1126/sciadv.aay6721

68. Crewe C, Joffin N, Rutkowski JM, Kim M, Zhang F, Towler DA, et al. An endothelial-to-adipocyte extracellular vesicle axis governed by metabolic state. Cell. (2018) 175:695-708.e613. doi: 10.1016/j.cell.2018.09.005

69. Hartwig S, De Filippo E, Göddeke S, Knebel B, Kotzka J, Al-Hasani H, et al. Exosomal proteins constitute an essential part of the human adipose tissue secretome. Biochim Biophys Acta BBA Proteins Proteomics. (2019) 1867:140172. doi: 10.1016/j.bbapap.2018.11.009

70. Valadi H, Ekström K, Bossios A, Sjöstrand M, Lee JJ, Lötvall JO. Exosomemediated transfer of mRNAs and microRNAs is a novel mechanism of genetic exchange between cells. Nat Cell Biol. (2007) 9:654-9. doi: 10.1038/ncb1596

71. Hawari FI, Rouhani FN, Cui X, Yu Z-X, Buckley C, Kaler M, et al. Release of full-length 55-kDa TNF receptor 1 in exosome-like vesicles: a mechanism for generation of soluble cytokine receptors. Proc Nat Acad Sci. (2004) 101:1297-302. doi: 10.1073/pnas.0307981100

72. Wang Y-c, Li Y, Wang X-y, Zhang D, Zhang $H$, Wu Q, et al. Circulating miR-130b mediates metabolic crosstalk between fat and muscle in overweight/obesity. Diabetologia. (2013) 56:2275-85. doi: 10.1007/s00125-013-2996-8

73. Yu Y, Du H, Wei S, Feng L, Li J, Yao F, et al. Adipocyte-derived exosomal MiR27a induces insulin resistance in skeletal muscle through repression of PPAR $\gamma$. Theranostics. (2018) 8:2171. doi: 10.7150/thno.22565

74. Gao H, Luo Z, Jin Z, Ji Y, Ying W. Adipose tissue macrophages orchestrate $\beta$ cell adaptation in obesity through secreting miRNA-containing extracellular vesicles. bioRxiv [Preprint]. (2020). doi: 10.1101/2020.06.12.148809

75. Sano S, Izumi Y, Yamaguchi T, Yamazaki T, Tanaka M, Shiota M, et al. Lipid synthesis is promoted by hypoxic adipocyte-derived exosomes in 3T3-L1 cells. Biochem Biophys Res Commun. (2014) 445:327-33. doi: 10.1016/j.bbrc.2014.01.183

76. Liu Z, Gan L, Zhang T, Ren Q, Sun C. Melatonin alleviates adipose inflammation through elevating $\alpha$-ketoglutarate and diverting adiposederived exosomes to macrophages in mice. J Pineal Res. (2018) 64:e12455 doi: 10.1111/jpi.12455

77. Arner P, Kulyté A. MicroRNA regulatory networks in human adipose tissue and obesity. Nat Rev Endocrinol. (2015) 11:276. doi: 10.1038/nrendo.2015.25

78. Ha M, Kim VN. Regulation of microRNA biogenesis. Nat Rev Mol Cell Biol. (2014) 15:509-24. doi: 10.1038/nrm3838

79. Geng L, Lam KS, Xu A. The therapeutic potential of FGF21 in metabolic diseases: from bench to clinic. Nat Rev Endocrinol. (2020) 16:654-67. doi: 10.1038/s41574-020-0386-0

80. Liu T, Sun Y-C, Cheng P, Shao H-G. Adipose tissue macrophage-derived exosomal miR-29a regulates obesity-associated insulin resistance. Biochem Biophys Res Commun. (2019) 515:352-8. doi: 10.1016/j.bbrc.2019.05.113

81. Zhang Y, Song K, Qi G, Yan R, Yang Y, Li Y, et al. Adipose-derived exosomal miR-210/92a cluster inhibits adipose browning via the FGFR1 signaling pathway in high-altitude hypoxia. Sci Rep. (2020) 10:1-15. doi: 10.1038/s41598-020-71345-8

82. Huang $\mathrm{J}-\mathrm{H}, \mathrm{Fu} \mathrm{C}-\mathrm{H}, \mathrm{Xu} \mathrm{Y}$, Yin $\mathrm{X}-\mathrm{M}$, Cao Y, Lin F-Y. Extracellular vesicles derived from epidural fat-mesenchymal stem cells attenuate NLRP3 inflammasome activation and improve functional recovery after spinal cord injury. Neurochem Res. (2020) 45:760-71. doi: 10.1007/s11064-019-02950-x

83. Yu C, Chen P, Xu J, Liu Y, Li H, Wang L, et al. hADSCs derived extracellular vesicles inhibit NLRP3inflammasome activation and dry eye. Sci Rep. (2020) 10:1-12. doi: 10.1038/s41598-020-71337-8 
84. Wen D, Qiao P, Wang L. Circulating microRNA-223 as a potential biomarker for obesity. Obes Res Clin Pract. (2015) 9:398-404. doi: 10.1016/j.orcp.2015.01.006

85. Haneklaus M, Gerlic M, Kurowska-Stolarska M, Rainey A-A, Pich D, McInnes IB, et al. Cutting edge: miR-223 and EBV miR-BART15 regulate the NLRP3 inflammasome and IL-1 $\beta$ production. J Immunol. (2012) 189:3795-9. doi: 10.4049/jimmunol.1200312

86. Ogawa R, Tanaka C, Sato M, Nagasaki H, Sugimura K, Okumura K, et al. Adipocyte-derived microvesicles contain RNA that is transported into macrophages and might be secreted into blood circulation. Biochem Biophys Res Commun. (2010) 398:723-9. doi: 10.1016/j.bbrc.2010.07.008

87. Ferrante SC, Nadler EP, Pillai DK, Hubal MJ, Wang Z, Wang JM, et al. Adipocyte-derived exosomal miRNAs: a novel mechanism for obesity-related disease. Pediatr Res. (2015) 77:447-54. doi: 10.1038/pr.2014.202

88. Alvarez-Dominguez JR, Bai Z, Xu D, Yuan B, Lo KA, Yoon MJ, et al. De novo reconstruction of adipose tissue transcriptomes reveals long non-coding RNA regulators of brown adipocyte development. Cell Metab. (2015) 21:764-76. doi: 10.1016/j.cmet.2015.04.003

89. Li P, Ruan X, Yang L, Kiesewetter K, Zhao Y, Luo H, et al. A liver-enriched long non-coding RNA, lncLSTR, regulates systemic lipid metabolism in mice. Cell Metab. (2015) 21:455-67. doi: 10.1016/j.cmet.2015.02.004

90. Gao J, Li X, Wang Y, Cao Y, Yao D, Sun L, et al. Adipocyte-derived extracellular vesicles modulate appetite and weight through mTOR signalling in the hypothalamus. Acta Physiol. (2020) 228:e13339. doi: 10.1111/apha.13339

91. Lee J-E, Moon P-G, Lee I-K, Baek M-C. Proteomic analysis of extracellular vesicles released by adipocytes of Otsuka Long-Evans Tokushima Fatty (OLETF) Rats. Prot J. (2015) 34:220-35. doi: 10.1007/s10930-015-9616-Z

92. Martínez MC, Andriantsitohaina R. Extracellular vesicles in metabolic syndrome. Circ Res. (2017) 120:1674-86. doi: 10.1161/CIRCRESAHA.117.309419

93. Kranendonk ME, Visseren FL, van Herwaarden JA, Nolte-'t Hoen EN, de Jager W, Wauben $\mathrm{MH}$, et al. Effect of extracellular vesicles of human adipose tissue on insulin signaling in liver and muscle cells. Obesity. (2014) 22:2216-23. doi: $10.1002 /$ oby.20847

94. Wiklander OP, Brennan MÁ, Lötvall J, Breakefield XO, Andaloussi SE. Advances in therapeutic applications of extracellular vesicles. Sci Transl Med. (2019) 11:eaav8521. doi: 10.1126/scitranslmed.aav8521

95. Andaloussi SE, Mäger I, Breakefield XO, Wood MJ. Extracellular vesicles: biology and emerging therapeutic opportunities. Nat Rev Drug Discov. (2013) 12:347-57. doi: 10.1038/nrd3978

96. Ntolios P, Manoloudi E, Tzouvelekis A, Bouros E, Steiropoulos P, Anevlavis S, et al. Longitudinal outcomes of patients enrolled in a phase Ib clinical trial of the adipose-derived stromal cells-stromal vascular fraction in idiopathic pulmonary fibrosis. Clin Respir J. (2018) 12:2084-9. doi: 10.1111/crj. 12777

97. Philandrianos C, Serrero M, Grimaud F, Magalon J, Visée C, Velier M, et al. First clinical case report of local microinjection of autologous fat and adipose-derived stromal vascular fraction for perianal fistula in Crohn's disease. Stem Cell Res Ther. (2018) 9:1-6. doi: 10.1186/s13287-017-0736-6

98. Fu T, Choi S-E, Kim D-H, Seok S, Suino-Powell KM, Xu HE, et al. Aberrantly elevated microRNA-34a in obesity attenuates hepatic responses to FGF19 by targeting a membrane coreceptor $\beta$-Klotho. Proc Nat Acad Sci. (2012) 109:16137-42. doi: 10.1073/pnas. 1205951109

Conflict of Interest: The authors declare that the research was conducted in the absence of any commercial or financial relationships that could be construed as a potential conflict of interest.

Copyright (C) 2021 Huang and Xu. This is an open-access article distributed under the terms of the Creative Commons Attribution License (CC BY). The use, distribution or reproduction in other forums is permitted, provided the original author(s) and the copyright owner(s) are credited and that the original publication in this journal is cited, in accordance with accepted academic practice. No use, distribution or reproduction is permitted which does not comply with these terms. 